\title{
Design of OTA-C Active Low Pass Filter Using Multiple OTA's
}

\author{
${ }^{1}$ Rajeshwari S. Mathad, ${ }^{2}$ M. M. Mutsaddi, ${ }^{3}$ S. V. Halse \\ ${ }^{I}$ Department of Electronics Basaveshwar Science College, BAGALKOT, India. \\ ${ }^{2}$ Department of Physics Basaveshwar Science College, Bagalkot, India \\ ${ }^{3}$ Department of Electronics,Karnataka State Women's University Bijapur, India
}

\begin{abstract}
The studies are carried out on OTA-C active filter using single OTA-C and multiple OTA-C filter. The multiple OTA-C filters have the advantage of tuning the floating inductor resulting in enhancement of gain and increase in cut-off frequency with increase in bias current. Different cases of tuning transconductance $g_{m l}$, $g_{m 1}, g_{m}$, and $g_{m 2}$ by changing the bias currents of multiple OTA 's are studied and results are discussed. The only one method of setting of $g_{m 1} g_{m 1}$ greater than $g_{m} g_{m 2}$ is an innovative in changing the cut off frequency as we have flexible tuning transconductances of $g_{m l}, g_{m 1}, g_{m}$ and $g_{m 2}$. Results are consistent and are encouraging in respect of tuning of transconductance with bias current under the condition of $g_{m 1} g_{m 1}$ greater than $g_{m} g_{m 2}$. However an OTA-C active filter other than the studied criteria has the limitations in designing of filters.
\end{abstract}

Keywords: OTA Operational Transconductance Amplifier OTA-C Operational Transconductance AmplifierCapacitance

\section{Introduction}

Limitations of passive filter studies are completely eliminated in active filter studies. However voltage controlled Op-Amp and current controlled OTA are considered as basic active elements in the design of active filters. The active filter design using op amp has a serious limitation over the applications in the high frequency regions. To overcome these limitations active filters using OTA are popular due to the salient features of OTA such as , the adjustable $g_{m}$ over wide range of bias current, excellent matching between amplifiers, the linearity of transconductance with bias current, controlled impedance buffers and high output signal to noise ratio, which popularises OTA in active filter design. In this paper first order active low pass filter using OTAs and $\mathrm{C}$ is explained in order to use the linearity of transconductance of OTA with bias current $[1,8,9]$.

Electronically tuneable circuits attracted considerable attention in the design of analog integrated circuits because different values of resistance, inductance capacitance can be obtained by the same device. Programmability is one the most attractive features of the Operational Transconductance Amplifier (OTA), since it possible to tune analog devices such as; filters, oscillators etc, electronically. OTA is a differential voltage controlled current source (VCCS) where the output current is controlled by an applied input voltage signal. This tunability is obtained by varying the transconductance $\left(\mathrm{g}_{\mathrm{m}}\right)$ of the OTA which in turn is controlled by the bias current or voltage. However, tunability is restricted by the limited bandwidth of $\mathrm{g}_{\mathrm{m}}$, which depends on the bias current. OTA is a commercially available active component which has been used widely in many applications. OTA circuits have been shown to be potentially advantageous for the design of many first and second order high-frequency analog filters. To achieve any required cut-off frequency in passive filter, we require different values of resistors \& inductors as calculated from mathematical expression; which may or may not be available in the market. To solve this problem, we can use OTAs. We achieve the required resistance and inductance values by varying its $g_{\mathrm{m}}$. The OTA's can be connected to design active elements, simulating the property of an inductor in order to be an alternative choice of an on-chip inductor instead of a passive spiral inductor which usually has low Q-factor and also not tunable. These elements are called the OTA-based inductors, grounded and floating [3-9].

A number of different OTAs are available in market from various manufacturers. With the use of LM13600 and CA3080 OTA's it is possible to achieve extremely linear transconductance characteristic with respect to amplifier bias current.

LM13600 consists of two current controlled transconductance amplifiers each with differential inputs \& push pull outputs. The two amplifiers shares common supplies but operate independently. Linearalizing diodes are provided at the inputs to reduce distortion and allow higher levels. The result is a $10 \mathrm{~dB}$ signal to noise improvement referred to $0.5 \%$. Controlled impedance buffers which are especially designed to compliment the dynamic range of the amplifier are provided.

OTA LM 13600 has the following features which make it suitable in the design of active filters.

1) $\mathrm{g}_{\mathrm{m}}$ adjustable over wide range and is linear

2) Excellent matching between amplifiers.

3) Linearising diodes

4) Controlled impedances buffers 
5) High output signal to noise ratio.

These featured are taken into consideration in studying OTA active filters. The practical applications of OTA-C filter is given in the studies of low pass filter, high pass filter and band pass filters. Since the device is current controlled transconductance amplifier, the filter exhibits various enhanced range of pass band and cut off frequency as shown by the observations $[10,11]$.

\subsection{OTA Fundamentals}

The OTA is a transconductance device in which the input voltage controls the output current. The transconductance gm makes the OTA as voltage controlled current source; whereas the op-amps are voltage controlled voltage source [1].

An ideal OTA is defined by Io $=g_{m}$ (Vin+ - Vin-) where the input and output resistances are infinite. The transconductance $\mathrm{g}_{\mathrm{m}}$ is given by:

$$
\mathrm{g}_{\mathrm{m}}=\frac{1}{2 \mathrm{VT}} \mathrm{I}_{\text {bias }}
$$

where $\mathrm{V}_{\mathrm{T}}$ : thermal voltage $=26 \mathrm{mV}$ at room temperature.

$\mathrm{I}_{\text {bias }}$ : bias current. The transconductance gm is directly proportional to bias current $\mathrm{I}_{\text {bias }}$

Characteristics of Ideal OTA can be summarized as follows,

Input impedance $(\mathrm{Zin})=\infty$

Output Impedance $(\mathrm{Z} 0)=\infty$

Inverting input current $\mathrm{I}_{0^{-}}=$Non-inverting input current $\mathrm{I}_{\mathrm{O}^{+}}$

Bandwidth $=\infty$

The Figures $1 \& 2$ shows the basic schematic and equivalent circuit of OTA.

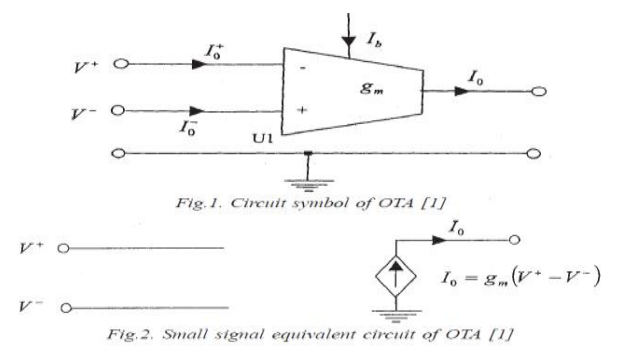

Circuit symbol of OTA can also be represented as shown in below figure

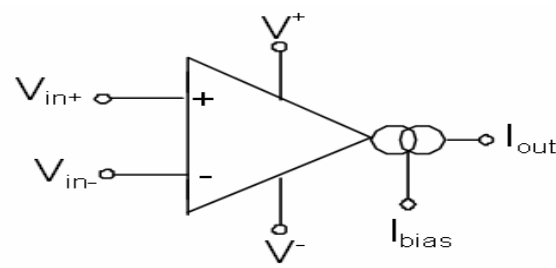

\subsection{Floating Inductor}

Floating inductors which are difficult to simulate with op-amps are very easy to be simulated by the use of OTA. The only task is to lift the inverting gm1 terminal of fig. 3 off the ground and device a circuit that generates $\mathrm{I}_{2}=\mathrm{I}_{1}$ at the second impedance terminal.

Fig. 8 shows a simple method, which needs only one additional OTA.

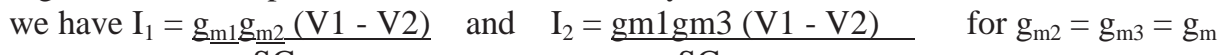

$$
\mathrm{SC} \quad \mathrm{SC}
$$

Floating inductor of value $: \mathrm{L}=\mathrm{C} / \mathrm{g}_{\mathrm{m} 1} \mathrm{~g}_{\mathrm{m}}$ has been simulated.

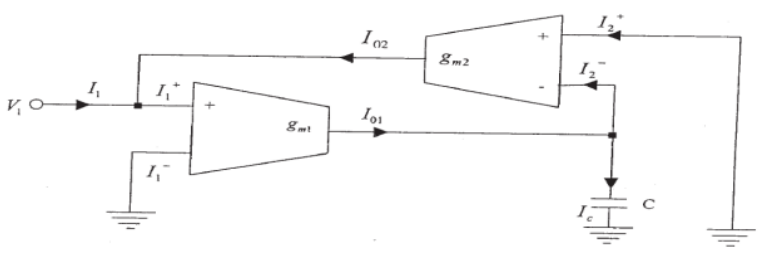

Fig 3 Simulation of floating inductor

\subsection{Derivation of OTA-C Filters from Passive Filters}

First order low pass filter. Consider a first order low pass filter as shown in fig. 3 below 


$$
\begin{aligned}
& \text { Vin }=\mathrm{I}[\mathrm{SL}+\mathrm{R}] \text { and } \mathrm{Vo}=\mathrm{IR} \\
& \text { Vo R/L } \\
& ---=\mathrm{T}(\mathrm{s})= \\
& \text { Vin } S+R / L
\end{aligned}
$$

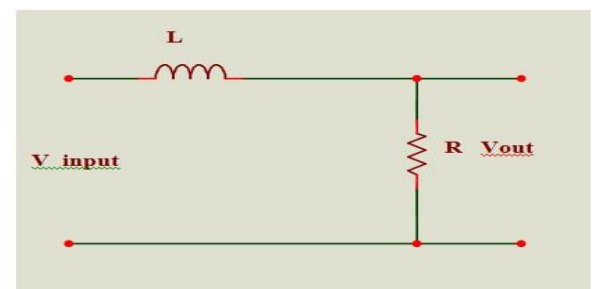

Fig. 4 Circuit diagram of passive Low Pass filter

\subsection{Realization using single OTA-}

From below figure we get

$\mathrm{I}_{1}=\mathrm{g}_{\mathrm{m}}(\mathrm{Vin}-\mathrm{V} 0)$

From above equation, the transfer function for considered filter is given by

$$
\mathrm{T}(\mathrm{s})=\frac{\mathrm{g}_{\mathrm{m}} / \mathrm{C}}{-\mathrm{S}+\mathrm{g}_{\mathrm{m}} / \mathrm{C}}
$$

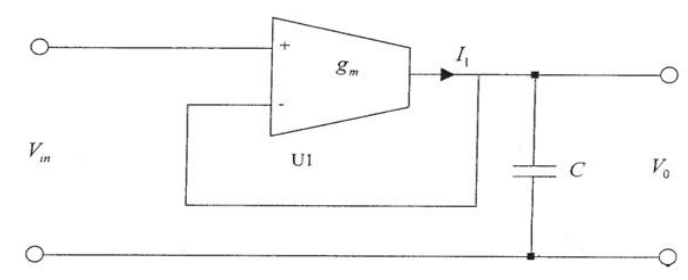

Fig-5 First order active low pass filter using single OTA (First method)

\subsection{Realization using multiple OTAs}

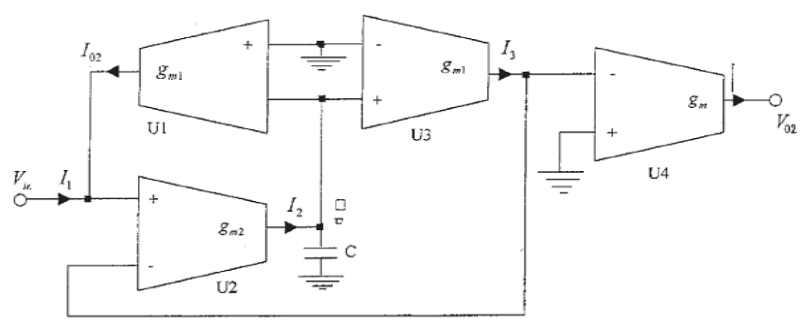

Fig-6 First order active low pass filter using multiple OTA (second method)

From above figure it can be inferred that

$\mathrm{V}_{3}=\mathrm{I}_{2} / \mathrm{SC}$

$\mathrm{I}_{2}=\left(\right.$ Vin $\left.-\mathrm{V}_{0}\right) \mathrm{g}_{\mathrm{m} 2}$

(Vin - Vo) $g_{\mathrm{m} 2}$

Solving above equations we have $\mathrm{V}_{3}=-\frac{\mathrm{C}}{\mathrm{O}}$

$\mathrm{I}_{4}=-\mathrm{g}_{\mathrm{m}} \mathrm{Vo}_{\mathrm{o}}$

$\mathrm{I}_{3}=-\mathrm{V}_{3} \mathrm{~g}_{\mathrm{m} 1}$

But we see that $\mathrm{I}_{3}+\mathrm{I}_{4}=0$

Therefore transfer function of the designed filter is

$$
\frac{\mathrm{V} 0}{\text { Vin }}=\mathrm{T}(\mathrm{s})=\frac{\mathrm{g}_{\mathrm{m} 1} \mathrm{~g}_{\mathrm{m} 2} / \mathrm{Cg}_{\mathrm{m}}}{\mathrm{S}+\left(\mathrm{g}_{\mathrm{m} 1} \mathrm{~g}_{\mathrm{m} 2} / \mathrm{Cg}_{\mathrm{m}}\right)}
$$

The above discussion states that we can achieve a tuneable range of cut off frequencies

The advantage of using single OTA and multiple OTAs can be summarized as follows:

1. In case of first method, by using single OTA We can change cut-off frequency by changing $g m$ but the degree of freedom for changing $\mathrm{g}_{\mathrm{m}}$ is only one. This will reflect change in value of floating inductance.

2. In case of second method, by using multiple OTAs cut-off frequencies can be changed by tuning $\mathrm{g}_{\mathrm{m} 1} \mathrm{~g}_{\mathrm{m} 2}$ or $\mathrm{g}_{\mathrm{m}}$ separately or in combination. So we have a larger tuneable floating inductance than the first one [2] 


\section{Experimental Setup And Results}

The stated circuit in "Fig 7" is simulated using Proteus professional 7. Same circuit is arranged on bread board using usual method, the output of the filter is measured. In first order low pass filter four OTAs with transconductance $\mathrm{g}_{\mathrm{m} 1} \mathrm{~g}_{\mathrm{m} 1}, \mathrm{~g}_{\mathrm{m} 2} \mathrm{~g}_{\mathrm{m}}$ are used. The transconductance can be tuned from bias current in different cases of their magnitudes.

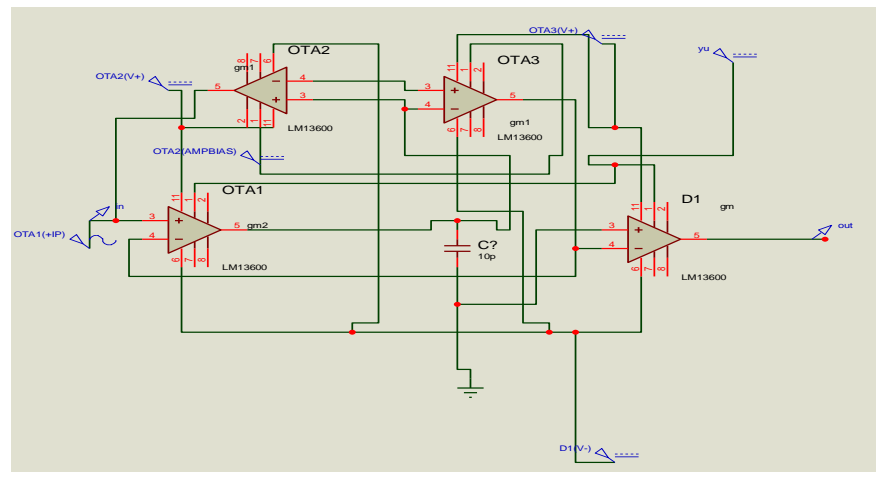

Fig. 7 Circuit diagram of OTA- C Low Pass filter

2.1Case-1: The bias current of $g_{\mathrm{m} 1} g_{\mathrm{m} 1}$ is equal to bias current of $\mathrm{g}_{\mathrm{m}} \mathrm{g}_{\mathrm{m} 2}$.

2.2Case-2: The bias current of $g_{\mathrm{m} 1} g_{\mathrm{m} 1}$ is greater than bias current of $\mathrm{g}_{\mathrm{m}} \mathrm{g}_{\mathrm{m} 2}$.

2.3Case-3: The bias current of $\mathrm{g}_{\mathrm{m} 1} \mathrm{~g}_{\mathrm{m} 1}$ is less than bias current of $\mathrm{g}_{\mathrm{m}} \mathrm{g}_{\mathrm{m} 2}$.

The stated circuits in "Fig 4" are simulated using Proteus professional 7. Same circuit is arranged on bread board using usual method, the output of the filter is measured.

In OTA-C low pass filter shown in "Fig-7" the results obtained for case-1, case-2 and case-3 are as follows.

For case-1 The bias current of $g_{\mathrm{m} 1} g_{\mathrm{m} 1}$ is equal to bias current of $\mathrm{g}_{\mathrm{m}} \mathrm{g}_{\mathrm{m} 2}$

Results are obtained only for bias current of $g_{\mathrm{m} 1} g_{\mathrm{m} 1=} g_{\mathrm{m}} \mathrm{g}_{\mathrm{m} 2}=5 \mathrm{~mA}$. For this bias current maximum gain or $\mathrm{G}_{\max }$ obtained is of order $-2.59 \mathrm{~dB}$ and at $-10 \mathrm{~dB}$ of gain, frequency is of order $18 \mathrm{MHz}$. For bias current of $\mathrm{g}_{\mathrm{ml}} \mathrm{g}_{\mathrm{ml}}=\mathrm{g}_{\mathrm{m}} \mathrm{g}_{\mathrm{m} 2}=10 \mathrm{~mA}$ maximum gain or $\mathrm{G}_{\mathrm{max}}$ obtained is of order $-2.96 \mathrm{~dB}$ and at $-10 \mathrm{~dB}$ of gain, frequency is of order $27 \mathrm{MHz}$. For bias current of $\mathrm{g}_{\mathrm{m} 1} \mathrm{~g}_{\mathrm{m} 1=} \mathrm{g}_{\mathrm{m}} \mathrm{g}_{\mathrm{m} 2}=11 \mathrm{~mA}$ maximum gain or $\mathrm{G}_{\max }$ obtained is of order $-4.67 \mathrm{~dB}$ and at $-10 \mathrm{~dB}$ of gain, frequency is of order $20.8 \mathrm{MHz}$.For any bias current above $11 \mathrm{~mA}$ gain goes on decreasing. Finally as bias current increases above $10 \mathrm{~mA}$ maximum gain or $\mathrm{G}_{\max }$ decreases with decreased cut off frequency.

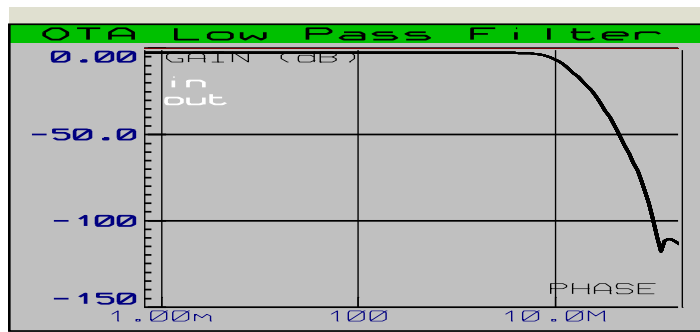

Fig. 8 Frequency response of Proteus professional 7 simulated OTA Low pass filter circuit, with pass band up to $18 \mathrm{MHz}$ at $-10 \mathrm{~dB}$ of gain. Maximum gain is $2.59 \mathrm{~dB}$, For bias current of $5 \mathrm{~mA}\left(\mathrm{i}, \mathrm{e} \mathrm{g}_{\mathrm{m} 1} \mathrm{~g}_{\mathrm{ml}}=\mathrm{g}_{\mathrm{m}} \mathrm{g}_{\mathrm{m} 2}=5 \mathrm{~mA}\right)$

For case-2 The bias current of $g_{\mathrm{m} 1} g_{\mathrm{m} 1}$ is greater than bias current of $\mathrm{g}_{\mathrm{m}} \mathrm{g}_{\mathrm{m} 2}$

For different combinations of $g_{m 1} g_{m 1}$ greater than $g_{m} g_{m 2}$ we can conclude that, if the difference between the bias currents is of smaller $\mathrm{G}_{\max }$ is also less, its around $0 \mathrm{~dB}$, and difference between band width or cut off frequencies is also less.

For example, if $\mathrm{g}_{\mathrm{m} 1} \mathrm{~g}_{\mathrm{m} 1}=15 \mathrm{~mA}$ and $\mathrm{g}_{\mathrm{m}} \mathrm{g}_{\mathrm{m} 2}=12 \mathrm{~mA}$ maximum gain or $\mathrm{G}_{\max }$ is $0 \mathrm{~dB}$ and at $-10 \mathrm{~dB}$ of gain ,frequency is of order $78 \mathrm{MHz}$. If $\mathrm{g}_{\mathrm{m} 1} \mathrm{~g}_{\mathrm{m} 1}=10 \mathrm{~mA}$ and $\mathrm{g}_{\mathrm{m}} \mathrm{g}_{\mathrm{m} 2}=8 \mathrm{~mA}$ maximum gain is $2.33 \mathrm{~dB}$ and at $-10 \mathrm{~dB}$ of gain, frequency is of order $97 \mathrm{MHz}$.

If the difference between the bias currents is of larger value gain enhances and pass band width also increases. Which is more advantageous in tuning the value of floating inductance floating inductance?

For example, if $\mathrm{g}_{\mathrm{m} 1} \mathrm{~g}_{\mathrm{m} 1}=10 \mathrm{~mA}$ and $\mathrm{g}_{\mathrm{m}} \mathrm{g}_{\mathrm{m} 2}=5 \mathrm{~mA}, \mathrm{G}_{\mathrm{max}}$ or maximum gain is $2 \mathrm{~dB}$ and at $-10 \mathrm{~dB}$ of gain, frequency is of order $121 \mathrm{MHz}$. If $\mathrm{g}_{\mathrm{m} 1} \mathrm{~g}_{\mathrm{m} 1}=15 \mathrm{~mA}$ and $\mathrm{g}_{\mathrm{m}} \mathrm{g}_{\mathrm{m} 2}=10 \mathrm{~mA}$ maximum gain or $\mathrm{G}_{\max }$ is $4.13 \mathrm{~dB}$ and at $10 \mathrm{~dB}$ of gain, frequency is of order $175 \mathrm{MHz}$. If $\mathrm{g}_{\mathrm{m} 1} \mathrm{~g}_{\mathrm{m} 1}=20 \mathrm{~mA}$ and $\mathrm{g}_{\mathrm{m}} \mathrm{g}_{\mathrm{m} 2}=10 \mathrm{~mA}, \mathrm{G}_{\max }$ or maximum gain is $6.16 \mathrm{~dB}$ and at $-10 \mathrm{~dB}$ of gain , frequency is of order $270 \mathrm{MHz}$. 


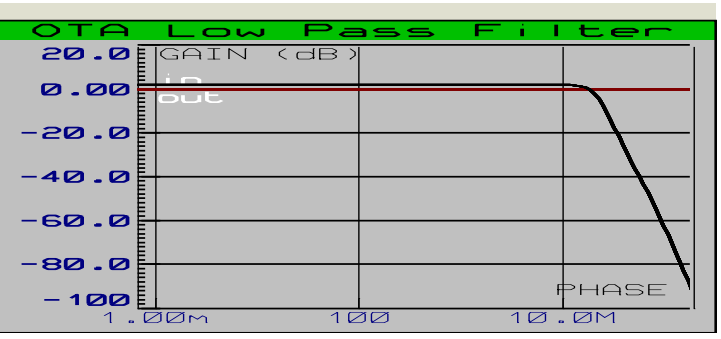

Fig. 8 Frequency response of Proteus professional 7 simulated OTA Low pass filter circuit, with pass band up to $121 \mathrm{MHz}$ at $-10 \mathrm{~dB}$ of gain. Maximum gain is $2 \mathrm{~dB}$ for bias current of $\mathrm{g}_{\mathrm{m} 1} \mathrm{~g}_{\mathrm{m} 1}=10 \mathrm{~mA}$ and $\mathrm{g}_{\mathrm{m}} \mathrm{g}_{\mathrm{m} 2}=5 \mathrm{~mA}$

For case -3: The bias current of $g_{\mathrm{m} 1} g_{\mathrm{m} 1}$ is less than bias current of $\mathrm{g}_{\mathrm{m}} \mathrm{g}_{\mathrm{m} 2}$ results are absurd.

\section{Conclusion}

OTA-C filter realized with minimum number of OTA's and capacitors is a innovative method of designing active filters which have flexibility in respect of the gain and pass band width. The above discussion concludes that we can achieve a tuneable range of cut off frequencies only by changing bias current which changes parameter $\mathrm{g}_{\mathrm{m}}$ transconductance of a device.

The advantage of using single OTA and multiple OTAs can be summarized as follows:

1. In case of first method, by using single OTA, we can change cut-off frequency by changing $\mathrm{g}_{\mathrm{m}}$ but the degree of freedom for changing $\mathrm{g}_{\mathrm{m}}$ is only one.

2. In case of second method, by using multiple OTAs cut-off frequency can be changed by tuning $\mathrm{g}_{\mathrm{m} 1}, \mathrm{~g}_{\mathrm{m} 2}$ or $\mathrm{g}_{\mathrm{m}}$ separately or in combination. So we have larger tuneable parameters than the first method.

The studied low pass filter exposes the salient features of low pass filter without feedback arrangement for low bias current. The circuit has limitations with respect to the values of $g_{\mathrm{m} 1} g_{\mathrm{m} 1}$ and $\mathrm{g}_{\mathrm{m}} \mathrm{g}_{\mathrm{m} 2}$. The results are encouraging in respect of bias current having magnitudes greater than $g_{\mathrm{m}} \mathrm{g}_{\mathrm{m} 2}$, (i,e $\mathrm{g}_{\mathrm{m} 1} \mathrm{~g}_{\mathrm{m} 1}$ greater than $\mathrm{g}_{\mathrm{m}} \mathrm{g}_{\mathrm{m} 2}$ ) where the enhancement in the gain and in the cut off frequency is observed, where as in other cases the results are not encouraging in obtaining the values of cut off frequencies in the design of OTA-C low pass filter, due to nonlinearity of transconductance $\mathrm{g}_{\mathrm{m}} \mathrm{g}_{\mathrm{m} 2}$.

The Low Pass filter structure using OTA has a several advantages in high frequency applications. The structure is very simple and easy to design. No external resistor is required, which can save the area in case of fabricating in a silicon chip. Moreover, the cut off frequency can be tuned electronically by adjusting the bias current of OTAs. These filters are used in high frequency mixtures and in medical applications where conventional op-amp filters can't be used in high frequency application due to their limited gain bandwidth product [1-14].

\section{Acknowledgements}

We are very much thankful to Prasant K. Mahapatra and et al for the design idea which has been taken from the paper "Realization of Active Filters Using Operational Transconductance Amplifier (OTA)" J. Instrum. Soc. India 35(1) 1-9 and encouraged for further work.

\section{References}

[1] Achim Gratz "Operational Transconductance Amplifiers” http://Synth.Stromeko.net/diy/OTA.pdf.

[2] Prasant K. Mahapatra, Manjeet Singh and Neelesh Kumar "Realization of Active Filters Using Operational Transconductance Amplifier (OTA)" J. Instrum. Soc. India 35(1) 1-9

[3] Neha Gupta , Meenakshi Suthar, Sapna Singh, Priyanka Soni “Active Filter Design Using Two OTA based Floating Inductance Simulator” International Journal of VLSI \& Signal Processing Applications, Vol.2,Issue 1, Feb 2012, (47-50), ISSN 2231-3133 ( Online)

[4] Hwang.Y.S, Liu.S.I, Wu.D.S, Wu.Y.P "Table_based linear transformation filter using OTA-C techniques" Electronics LETTERS vol.30 No.24 pp,1-2., 1994 .

[5] A.M Soliman, “A Grounded Inductance Simulation Using the DVCCS/DVCVS”, Proc. IEEE,Vol. 66 pp 1089-1091, Sep 1978.

[6] Y. Sun, Design of high frequency integrated analogue filters. London:IEE Press, 2002

[7] A.M Soliman, "A Grounded Inductance Simulation Using the DVCCS/DVCVS", Proc. IEEE,Vol. 66 pp 1089-1091, Sep 1978.

[8] A. Thanachayanon, "CMOS Floating Active Inductor and Its Applications to Bandpass Filter and Oscillator Design," lEE Proc. Circuits Devices Syst. , vol. 147, 1EE, pp.. 42-48,2000.

[9] Koomagaew C, Petchmaneelumka W, Riewruja V, “OTA based Floating Inductance Simulator", ICCAS-SICE, pp 857-860, Aug. 2009.

[10] National Semiconductor, Application Note, LM 13600 Dual Operational Transconductance Amplifiers with Linearizing Diodes and Buffers", Feb. 1995

[11] Intersil Americas Inc., An IC Operational Transconductance Amplifier (OTA) with Power Capability, Oct. 2000. AN6077.3. (Review Article)

[12] Vasudev K. Aatre, "Network Theory \& Filter Design", 2nd Edition, New Age International (P) Ltd., 1980

[13] Schaumann et. Al., "Design of Analog Filters Passive, Active RC and Switched Capacitor" Prentice Hall, Englewood Cliff, New Jersy, 1990

[14] Sergio Franco, "Design with operational amplifiers and analog integrated circuits", 3rd ed, pp 133-141 